\title{
METABOLIC DIVERSITY IN PLANTS
}

Bhargava Neha* and Gomase V.S. ${ }^{+}$

"Padmashree Dr. D.Y. Patil University, Navi Mumbai, 400614, India, nehabhargava205@gmail.com

'School of Technology, S.R.T.M. University, Sub-Centre, Latur, 413512, India

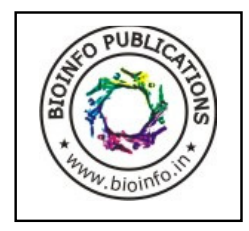

Abstract: Metabolic diversity in various plants of various kingdoms and species has successfully solved many fundamental questions arising due to different and varying growth conditions and parameters they are subjected to. It is important to study metabolic diversity to understand how science has evolved and succeeded to address the practical application of recombinant DNA technology for the benefit of plants themselves and also to know how these metabolic changes help them adapt to diverse conditions.

Keywords: Metabolic diversity, Transcriptome, Genome, Gene expression, Stress tolerance

\section{Introduction}

Metabolic changes underpin overall plant development and responses to stresses they are subjected to. It is also frequently found that metabolic information reflects biological endpoints more accurately than transcript information or protein analysis, whilst quality traits in many important plants are dependent on metabolic composition. The metabolome showcases a very important and critical aspect of a plant's physiology and growth characteristics. Thus ultimately, it represents its economic value. The science of metabolomics is very useful in assessing genotypic and phenotypic traits in plants and in defining biochemical changes associated with developmental changes during plant growth An extraordinary range of genetically distinct and metabolically diverse plants is displayed remarkably due to genetic manipulations.

Despite concerns regarding potential loss of genetic diversity through domestication, genetic resources still exist through wild and exotic germplasms. Additionally, emerging biotechnological approaches such as RNAi silencing and the transgenic modification of regulatory genes offer new and fascinating opportunities for enhancing diversity and eliciting trait improvements. Metabolomics, through comparative assessments of metabolic diversity in domesticated and nondomesticated plants, and through evaluations of the compositional impact of metabolic engineering efforts can support breeding programmes designed to elicit trait improvements [1].

The evolution of the ability to synthesize specialized metabolites is likely to have been key for survival and diversification of different plant species. For example, Oats (Avena spp.) produce antimicrobial triterpenoids (avenacins) that protect against disease. Although clusters of paralogous genes are common in plants (e.g., gene clusters for rRNA and specific disease resistance), reports of clusters of genes that do not share sequence relatedness and whose products contribute to a single selectable function are rare [2].

There are several 'mainstream' metabolic pathways that occur in a wide variety of living organisms. It is certainly important to note that metabolic processes in organisms other than man or animals may be quite different. For Example, (1) Photosynthesis ultimately enables plants to create all their carbon compounds from $\mathrm{CO} 2$ and water. However, even plants do perform catabolic reactions on endogenous macromolecules. (2) Plants accumulate an amazing diversity of phytochemicals that play important roles in the interaction of plants with the environment. A few mechanisms have described the evolution of phytochemicals from the perspective of the biosynthetic enzyme. The transcription factors that regulate these pathways have also evolved to ensure the coordinate expression of all the genes in a pathway [3].

\section{Genome-wide transcriptome analysis}

Transcriptome analysis is of growing importance in understanding how altered expression of genetic variants contributes to fight against complex diseases, environmental stresses or other attributes. Analysis of genome-wide differential RNA expression provides researchers with greater insights into biological pathways and molecular mechanisms that regulate cell fate, growth and development.

Whole-genome duplications provide the genetic material for evolutionary innovation. Ohno, stated that "natural selection merely modified while redundancy created". Since then, comparisons between whole-genome sequences have provided incontrovertible evidence that several plant genomes have undergone multiple ancient polyploidization events during their evolution [4].

Arabidopsis Pollen Transcriptome analysis The analysis of the entire genome of pollen transcriptome clearly shows the uniqueness of differentiation that sets apart the mature male gametophyte from the sporophyte. When the sporophyte transcriptome throughout its development and the pollen transcriptome were compared, it showed reduced complexity and a unique composition. The pollen transcriptome analysis revealed the over-representation of mRNAs encoding proteins involved in cell wall metabolism, cytoskeleton, and signalling and under-representation of mRNAs involved in transcription and protein synthesis [5]. 
Gene-expression and complexity of auxinregulated gene expression

The Auxin Response Factors (ARFs), modulating expression of auxin response genes and the Aux/IAA proteins, its negative regulators, regulate various auxin responses through auxin perception mediated by the Fbox proteins TIR1/AFBs. The transcriptome regulated downstream of $I A A 1$, a stabilized IAA1 mutant protein, was identified in order to study the regulatory mechanisms of Aux/IAAARF and expression analysis in various arf mutants suggested that all iaa1-regulated auxin-response genes are controlled by ARFs in a positive or a negative manner. In the contrary, it was found that ARFs did not play a critical role in down-regulation. This portrays the diversity and complexity of mechanisms of Aux/IAA-ARF- and auxin-regulated gene expression [6].

\section{Transcriptome analysis during cell division in plants}

The synchronized tobacco Bright Yellow-2 cells and cDNA-amplified fragment length polymorphism-based genome wide expression analysis was used to build a comprehensive collection of plant cell cycle-modulated genes. Approximately 1,340 genes were identified that are periodically expressed, including known cell cycle control genes, even the plant specific ones. Some unknown plant genes were homologous to cell cycle-regulatory genes of other organisms. Many of the genes encode novel or uncharacterized proteins which indicates that several processes underlying cell division are still largely unknown [7].

\section{Genome analysis in abiotic stress conditions in higher plants}

Environmental stresses represent the most limiting factors for agricultural productivity. Apart from biotic stress caused by plant pathogens, there are a number of abiotic stresses such as extremes in temperature, drought, salinity, heavy metals and radiation which all have detrimental effects on plant growth and yield. However, certain plant species and ecotypes have developed various mechanisms to adapt to such stress conditions [8]. Several genes that respond to these stresses are studied at the transcriptional level. Recent molecular and genetic studies have revealed the presence of many signalling components involved in the signalling pathways of these stresses. Furthermore, gene expression profiling using cDNA microarrays or gene chips has identified many genes that are regulated by drought-, cold-, or high-salinity stresses [9].

Freezing temperatures are a major factor limiting the geographical locations suitable for growing plants and periodically account for significant losses in plant productivity. Many plants increase in freezing tolerance upon exposure to low nonfreezing temperatures, a phenomenon known as "cold acclimation" which is known to include the expression of certain cold-induced genes that work to stabilize membranes against freeze-induced injury [10]. The products of the stress-inducible genes have been classified into two groups: those that directly protect against environmental stresses and those that regulate gene expression and signal transduction in the stress response [11].

While improving their resistance to stress, plant growth promoting Rhizobium (PGPR) can stimulate developmental changes in host plants, disrupt phytopathogen organization, induce systemic resistance to pathogens, affect phytohormone production, and improve nutrient and water management [12].

Transcriptome analysis of Induced systemic drought tolerance

The plant root colonization by Pseudomonas cholororaphis 06 can induce tolerance in Arabidopsis thaliana to drought. Root colonization in watered plants, have increased transcript accumulation from genes associated with defense, response to activated oxygen species, and auxin- and jasmonic acidresponsive gene but decreased transcription factors associated with ethylene and $A B A$ signaling. The root colonization in plants withholding water, have clusters of gene that were up-regulated in the $P$. chlororaphis O6colonized plants that were down-regulated in the noncolonized plants displaying drought stress and vice versa [13]. Thus, it is rightly shown that gene expression in plants lacking adequate water can be remarkably influenced by microbial colonization leading to plant protection.

\section{Function of rhizobial membrane lipids}

Biosyntheses and possible functions of membrane lipids in plant-associated bacteria

Due to their hydrophobic interior, cell membranes function as diffusion barriers for most hydrophilic substances, thus controlling the import or export of such substances by the means of specific proteins. Eubacterial membranes contain not only membrane proteins but also the so-called bilayer-forming membrane lipids, predominantly consisting of phospholipids, in some organisms and especially under certain physiological conditions, also of phosphorus-free membrane lipids. Membrane lipids in most bacteria generally consist of the glycerophospholipids phosphatidylglycerol, cardiolipin, and phosphatidylethanolamine (PE) [14].

\section{Symbiotic efficiency and acid tolerance}

Rhizobium tropici CIAT899 was observed to be highly tolerant to many environmental stresses and is a good competitor for nodule occupancy of common bean plants in acid soils. The genetic analysis of the locus disrupted by the Tn5 insertion in mutant 899-PV9 caused the 
discovery of sycA which is a homolog of the $\mathrm{CIC}$ family of chloride channels and $\mathrm{Cl} / \mathrm{H}+$ exchange transporters. Any nonpolar deletion in this gene caused serious deficiencies in nodule development, nodulation competitiveness, and N2 fixation on Phaseolus vulgaris plants, reason being its reduced ability to invade plant cells and to form stable symbiosomes.

A mutant carrying a nonpolar deletion in olsC, found downstream of sycA and homologous to aspartyl/asparaginyl beta-hydroxylases, is symbiotically defective, whereas overexpressed OlsC in the complemented strain provokes an acid-sensitive phenotype. Thus, a CIC homolog contributes to symbiotic performance and acid tolerance [15].

\section{Acidic growth conditions}

Lysyl-phosphatidylglycerol (LPG) is a commonly found membrane lipid in several gram-positive bacteria but is never heard to be seen in gram-negative bacteria. In Staphylococcus aureus, the gene product of mprF is responsible for LPG formation. The sequenced bacterial genome analysis reveals that genes coding for homologs of MprF from $S$. aureus are present in several classes of organisms throughout the bacterial kingdom. The expression of IpiA, low $\mathrm{pH}$ - inducible gene, from $R$. tropici in the heterologous hosts Escherichia coli and Sinorhizobium meliloti causes formation of LPG. LPG biosynthesis fails when IpiA is deleted and is restored upon complementation of IpiA-deficient mutants with a functional copy of the IpiA gene [16].

Biosynthesis of lipid A in Rhizobium leguminosarum

The most important character in an economical nitrogen-fixing bacterium is to be an aggressive root colonizer. In evaluating the root colonization capacity of rhizosphere bacteria, it is necessary to distinguish between adaptation to the rhizosphere and the ability to develop on, and keep pace with, developing roots [17].

Nitrogen-fixing symbiotic bacteria of the genus Rhizobium are of interest because they have a lipid A which is structurally distinct from that of enteric bacteria. The determinant of the nitrogen-fixing symbiosis with legumes in Rhizobium spp. is Lipopolysaccharides (LPS). LPS is anchored to the outer surface of the outer membrane by the lipid A moiety.

A number of notable structural differences exist between the lipid A of Escherichia coli and that of Rhizobium leguminosarum, indicating that diverse biosynthetic pathways may also exist. The early steps in lipid A biosynthesis pathway are conserved and the divergence leading to rhizobial lipid A may occur at a later stage in the pathway [18].

\section{Symbiotic interactions and nodule metabolism}

The fixation of molecular nitrogen into bioavailable compounds for cellular anabolism is a process restricted to some bacteria, termed as diazotrophs. The rhizobia-legume symbiosis is characterised by root-nodule formation of the plant host, which are colonised by the endosymbiotic rhizobia, called as bacteroids. This mutual symbiosis involve nodulated plant roots that supply the bacteria with energy-rich carbon compounds and obtain fixed nitrogen by the bacteroids in return.

Rhizobia-legume symbioses are not the only root-nodule forming interactions of bacteria and plants. Actinobacteria of the genus Frankia spp. are known to develop nodules for nitrogen fixation in various families and orders of angiosperms known as actinorhizal plants [19]. Rhizobium meliloti

The symbiosis between Rhizobium and legumes is highly specific. For example, R. meliloti elicits the formation of root nodules on alfalfa and not on vetch. $R$. meliloti nodulation (nod) genes determine the production of acylated and sulfated glucosamine oligosaccharide signals. The purified Nod factors (sulfated or not) from nodH+ or nodHstrains showed the same plant specificity in several bioassays viz., root hair deformations, nodulation, changes in root morphology, as the bacterial cells from which they were purified [20].

\section{Arbuscular Mycorrhizal Symbiosis}

The Arbuscular Mycorrhiza is unequivocally the world's most important symbiosis. It is of great mutual advantage bringing together the roots of over $80 \%$ of land plant species and fungi of the Phylum Glomeromycota. Host plants benefit from improved uptake of soil nutrients such as phosphate and nitrogen. Arbuscular mycorrhizal fungal hyphae take up both all forms of nitrogen, nitrate and ammonium (NO3and $\mathrm{NH} 4+$ ) from the soil [21].

Cyanobacterial associations with bryophytes

Cyanobacteria form a variety of associations with bryophytes ranging from the almost accidental to very close symbioses. Nostoc is a widespread cyanobacterial genus that is a common symbiont and the association between Nostoc and hornworts is an excellent example of a close symbiosis [22].

\section{Bradyrhizobium japonicum USDA110 in symbiosis with soybean}

Bradyrhizobium japonicum USDA110 is a gram negative soil bacterium that forms root nodules specifically on soybean. During nodule development, it undergoes together with morphological alteration of cells biochemical and physiological changes. Many studies have 
reported that a large number of proteins are expressed as specific or up-regulated in B. japoniucm bacteroids [23].

Responses of the host

\section{Periodical cicada oviposition damage}

Insect oviposition on plants is much known, but further studies involving response of host plants to oviposition damage are still pending. The patterns of oviposition vary spatially and temporally but it is found that ovipositing insects that portray outbreak characteristics may have strong effects on host plants during peak abundance. Particularly, periodical cicadas (Magicicada spp.) may reduce the performance of host plants when they synchronously grow in massive numbers to mate and oviposit on host plants. Oviposition densities on unnetted host plants were comparable to levels documented in other studies, which indicate that cicada oviposition damage did not generally reduce the performance of native or exotic host plants [24].

\section{Genome stability and methylation response} to environmental stress

Genome instability generally refers to the susceptibility of the genome to rearrangements and activation of mobile elements, whereby a stable genome impedes these mechanisms. This stability is largely due to the addition of functional groups, most commonly methyl groups, to DNA and/or histones. The loss of methyl groups, termed hypomethylation, allows for rearrangement events, such as homologous recombination, to occur. Homologous recombination acts both as a double-strand break repair mechanism and the mechanism underlying crossing over events during meiosis. Hypermethylation, the addition of methyl groups, together with specific histone modifications, stabilize the genome and prevent recombination events. However, the homologous recombination mechanism can prove dangerous in cells, as it can be responsible for the induction of recessive genotypes from heterozygous loci. Thus, genome stability must be closely monitored to balance this risk with the need for genome diversity [25].

Plant responses to environmental stress

Plants continuously respond to stimuli that alter their physiology, morphology and development throughout their life. Many of these stimuli (called as stresses) are of an external nature and have an adverse effect on growth, development and reproduction. But plants adapt to such conditions by developing certain traits suitable to a particular stress. Thus, they can survive but at a slow and gradual rate. Conversely, plants are able to acclimate on a reduced timescale by modifying their homeostasis and, therefore, adjusting to a frequently changing environment. The fact that plants can successfully react to unrelated physical, chemical or temporal environmental factors suggests the existence of complex perception and response signalling pathways [25].

Expression of the Arabidopsis $\mathrm{TCH}$ genes is markedly up regulated in response to a variety of environmental stimuli including the seemingly innocuous stimulus of touch. The mechanism(s) and factors that control $\mathrm{TCH}$ gene regulation are understood in order to enable plants to respond to environmental conditions. The $\mathrm{TCH}$ proteins are calmodulin, calmodulin-related proteins and a xyloglucan endotransglycosylase. Expression analyses and localization of protein accumulation indicates that the potential sites of $\mathrm{TCH}$ protein function include expanding cells and tissues under mechanical strain [26]

\section{Determining differential response}

In a study, disease severity was measured after 10, 20 and 30 days of plant growth. The pathogen and dual treatment ( $R$. solanacearum with $G$. mosseae) were not significantly different. Soil $\mathrm{pH}$ was greatly influencing the pathogen and AMF microbe. Glomus mosseae mycorrhizosphere was more alkaline ( $\mathrm{pH} 5.9)$ compared to the pathogen mycorrhizosphere (pH 4.9). The concentration of bacterial cell in the $R$. solancearum soil was not different from the dual treatment after 60 days of plant growth. Spore germination was influenced by the interaction between the soil pathogen and AMF. This provides an evidence about the role of plant host in increasing the spores germination influenced by many substances produced by the host root (root exudates) [27].

\section{Expression patterns \\ Novel patterns of gene expression in polyploid plants}

Genome doubling, or polyploidy, is a major factor accounting for duplicate genes found in most eukaryotic genomes. Polyploidy has considerable effects on duplicate gene expression, including silencing and up- or down regulation of one of the duplicated genes. These changes can arise with the onset of polyploidization or within several generations after polyploid formation and they can have epigenetic causal factors [28].

Patterns of gene action in plant development The crucifer Arabidopsis thaliana has been used widely as a model organism for the study of plant development. Efficient insertional mutagenesis system in Arabidopsis permits identification of genes by their patterns of expression during development. Transposable elements of the Ac/Ds system carrying the GUS reporter gene have been designed to act as enhancer traps or gene traps. Transposon insertion lines with specific GUS expression patterns provide valuable markers for studies of Arabidopsis development and identify new cell types or subtypes in plants. The diversity of gene expression patterns generated has 
suggested that the identification and cloning of Arabidopsis genes expressed in any developmental process is feasible using this system [29].

\section{Patterns of green fluorescent protein} expression in transgenic plants

Many modified forms of genes encoding green fluorescent protein (GFP) can be macroscopically detected when expressed in whole plants. This technology has opened up new uses for GFP such as monitoring transgene presence and expression in the environment once it is linked or fused to a gene of interest. It helps in whole-plant or wholeorgan GFP visualization is required [30].

\section{Native DNA transformation}

Crop genetic engineering relies on the introduction of foreign DNA into plant genomes. Native genes and regulatory elements can be reintroduced into plants without the need to use selectable markers. By also using transfer DNAs that are derived from within the targeted compatibility group, genetically engineered plants can now be produced that lack any foreign DNA [31]. DNA of another species can be integrated in a plant genome by natural processes, but the term "transgenic plants" refers to plants created in a laboratory using recombinant DNA technology. It helps in making plants by artificial insertion of genes from other plants or species or entirely different kingdom plants in order to obtain characteristics of interest.

\section{Agrobacterium mediated}

Plant transformation mediated by the soil plant pathogen Agrobacterium tumefaciens has become the most used method for plant transformation. A. tumefaciens naturally infects the wound sites in dicotyledonous plant causing the formation of the crown gall tumours. A. tumefaciens is capable to transfer a particular DNA segment (T-DNA) of the tumour-inducing ( $\mathrm{Ti}$ ) plasmid into the nucleus of infected cells where it is subsequently stable integrated into the host genome and transcribed, causing the crown gall disease. The tumour formation is a transformation process of plant cells resulted from transfer and integration of T-DNA and the subsequent expression of T-DNA genes. Secondly, the TDNA genes are transcribed only in plant cells and do not play any role during the transfer process and any foreign DNA placed between the T-DNA borders can be transferred to plant cell, no matter where it comes from [32].

\section{Virus mediated}

Foreign gene is cloned in the region of nonessential sequences within the viral genome. And the genome is then injected and delivered into the host by the virus.
Virus-based vectors offer a reasonable alternative to Agrobacterium as a tool for transient protein expression in plant cells because of their ability to replicate in host cells autonomously, move locally from cell to cell, and sometimes even move systematically from leaf to leaf. This can potentially result in the high yield of recombinant proteins [33].

Also, many viral infections are systemic so that gene can be introduced into all the cells in a plant. Vectors for transferring genes into plants are based on DNA or RNA molecules that naturally express their genetic information in plant cells. The replicative genomes of plant viruses are non- integrative vectors compared to those vectors based on T- DNA of $A$. Tumefaciens, which are integrative gene vectors [34].

\section{Chemical mediated gene transfer}

Compared with biological (viral vectors) and physical methods, the major advantages of synthetic vectors (or chemical methods) are their simplicity, ease of production, and relatively low toxicity. Many synthetic compounds have been developed since DEAEdextran was first used in transfection experiments more than 35 years ago. Rapid progress in developing more efficient synthetic vectors has led to successful DNA delivery into a variety of cell types in vitro and in vivo [35]. Chemicals like polyethylene glycol (PEG) and dextran sulphate induce DNA uptake into plant protoplasts. Calcium phosphate is also used to transfer DNA into cultured cells.

The PEG-mediated transformation is simple and efficient, allowing a simultaneous processing of many samples, and yields a transformed cell population with high survival and division rates. The method utilizes inexpensive supplies and equipments, and helps to overcome a hurdle of host range limitations of Agrobacterium-mediated transformation [36].

\section{Microinjection}

Microinjection techniques for plant protoplasts utilize a holding pipette for immobilizing the protoplast while an injection pipette is utilized to inject the macromolecule. In order to manipulate the protoplasts without damage, the protoplasts are cultured for from about 1 to 5 days before the injection is performed to allow for partial regeneration of the cell wall [37].

DNA is directly injected into plant protoplasts or cells (specifically into the nucleus or cytoplasm) using fine tipped $(0.5-1.0$ micrometer diameter) glass needle or micropipette.

\section{Electroporation}

Involves a pulse of high voltage applied to protoplasts/cells/ tissues to make transient pores in the plasma membrane which facilitates the uptake of foreign DNA. The cells are placed 
in a solution containing DNA and subjected to electrical shocks to cause holes in the membranes. The foreign DNA fragments enter through the holes into the cytoplasm and then to the nucleus [38].

\section{Particle gun/Particle bombardment}

Microprojectile bombardment employs highvelocity metal particles to deliver biologically active DNA into plant cells [39]. This method depends on the impact of heavy metal particles on target tissues and delivery of coated DNA on particles in passing. The particles are accelerated to sufficient velocity by highly pressurized inert gas, usually helium. Macroparticles made of gold, tungsten, or silver have been used for gene delivery through gene gun. Gas pressure, particle size, and dosing frequency are critical factors that determine penetration efficiency to the tissues, the degree of tissue injury, and overall gene transfer levels [40].

\section{Liposome mediated gene transfer or Lipofection \\ Liposomes consist of an aqueous core entrapped by one or more bilayers composed of natural or synthetic lipids. Liposomes composed of natural phospholipids are biologically inert and weakly immunogenic, and they possess low intrinsic toxicity. Gene encapsulation in liposomal vesicles allows condensation of DNA plasmid into a highly organized structure, and protects DNA against degradation during storage and in the systemic circulation of the gene encoding a therapeutic protein [41]. Thus, it can safely deliver DNA into the host cell.}

\section{Conclusion}

From past over 20 years, copious research on plant genome is studied. This article has thrown a spark on the various genes of plants and their modifications which lights up the path of very survival of these plants, to their benefit or as a whole to the diversity. This metabolic diversity ranging from a single plant to various kingdoms has helped the evolution of better genome structures and many new species. By far, a lot of researches have focussed on these ideas while a lot more is yet to be discovered.

\section{References}

[1] Qi X., Bakht S., Leggett M., et al. (2004) PNAS, 101(21), 8233-8238.

[2] Harrigan, George G., Martino-Catt, et al. (2007) Metabolomics, 3(3), 259272

[3] Metabolism course notes. Author: Michael Palmer, University of Waterloo.

http://watcut.uwaterloo.ca/webnotes/M etabolism/page-1.3.html.

[4] Thomas Thiel, Andreas Graner, Robbie Waugh, et al. (2009) BMC Evol Biol., 9: 209.
[5] David Honys and David Twell (2003) Plant Physiol., 132(2), 640-652.

[6] Dong Ju Lee, Jong Wha Park, Han Woo Lee, et al. (2009) J. Exp. Bot., 60 (13), 3935-3957.

[7] Peter Breyne, Rozemarijn Dreesen, Klaas Vandepoele. (2002) PNAS, 99(23), 14825-14830.

[8] Hirt Heribert, Shinozaki. (2004) Topics in current genetics.

[9] Margarete Baier, Andrea Kandlbinder, Karl-Josef Dietz, et al. (2004) Plant Responses to Abiotic Stress Topics in Current Genetics, 4, 271-308.

[10] Thomashow M. F. (1990) Annu. Rev. Plant Physiol. Plant Mol. Biol. 50, 571-599.

[11] Seki M., Kamei A., YamaguchiShinozaki K., Shinozaki K. (2003) Curr. Opin. Biotechnol. 14: 194-199.

[12] Essaid Ait Barka, Jerzy Nowak, and Christophe Clement. (2006) Applied and environmental microbiology, 72(11), 7246-7252.

[13] Han Song Hee, Cho Song Mi, Oh, Sang A, et al. (2009) American society of plant biologists, Plant Biology.

[14] Isabel M. López-Lara, Christian Sohlenkamp, and Geiger. (2003) MPMI, 16(7), 567-579.

[15] Rojas-Jiménez K, Sohlenkamp C, Geiger O, et al. (2005) Mol Plant Microbe Interact., 18 (11), 1175-1185.

[16] Christian Sohlenkamp, Kanaan A Galindo-Lagunas, Ziqiang Guan, et al. (2007) APS journal, 20(11), 14211430.

[17] Ran Lifshitz, Joseph W. Kloepper, Fran M. Scher, et al. (1986) Applied and environmental microbiology, 51(2), 251-255.

[18] Neil p. J. Price, Theresa M. Kelly, Christian R. H. Raetz. (1994) Journal Of Bacteriology, 176(15), 4646-4655.

[19] Christoph Kneip, Peter Lockhart, Christine Voß, et al. (2007) BMC Evolutionary Biology.

[20] http://www.biomedcentral.com/14712148/7/55.

[21] Roche P, Debellé $F$, Maillet $F$, et al. (1991). Cell, 67(6), 1131-1143.

[22] Tian Chunjie, Shachar-Hill, Yair. (2009) American society of plant biologists, Plant Biology.

[23] Australian National Botanic Gardens, Australian Government, http://www.anbg.gov.au.

[24] Nomura Mika, Arunothayanan Hatthay, Dao, Tan Van, et al. (2009) American society of plant biologists, Plant Biology.

[25] Luke Flory S. and Brett Mattingly W. (2008) Oecologia, Vol. 156, No. 3, Pages 649-656. 
[26] Alexander Boyko, Palak Kathiria, Franz J. Zemp, et al. (2007) Nucleic Acids Res., 35(5): 1714-1725.

[27] Braam J., Sistrunk M.L., Polisensky D.H., et al. (1997) Planta, 203: S35S41.

[28] Tahat M.M., Kamaruzaman , Sijam and Othman R.(2010) Plant Pathology Journal, 9(4), 198-207.

[29] Keith L. Adams and Jonathan F. Wendel. (2005) TRENDS in Genetics, 21(10), 539-543.

[30] Sundaresan V., Springer P., Volpe T., (1995) Genes \& Dev., 9, 1797-1810.

[31] Brian K. Harper and C. Neal Stewart. (2000) Plant Molecular Biology Reporter 18: Page: 141.

[32] Simplot Company J.R. (2004) Trends in Plant Science, 9(9), 457-464.

[33] Gustavo A. de la Riva, Joel GonzálezCabrera, Roberto Vázquez-Padrón, et al. (1998) EJB Electronic Journal of Biotechnology, 1, 3.

[34] Sang-Min Chung, Manjusha Vaidya and Tzvi Tzfira. (2006) Trends in plant science, 11(1), 1-4.

[35] Chawla H.S. (2002) Science Publishers, 376-377.
[36] Dexi Liu, Evelyn F. Chia, Hui Tian (2003) Chemical Methods for DNA Delivery: An Overview, Gene Delivery to Mammalian Cells: Volume 1: Nonviral Gene Transfer Techniques, Series: Methods in Molecular Biology, 245, 3-23.

[37] Jaideep Mathur and Csaba Koncz. PEG-Mediated Protoplast Transformation with Naked DNA, Methods in Molecular Biology, Vol. 82: Arabidopsis Protocols, 267-276.

[38] Crossway Anne, Facciotti Daniel (1988) Plant cell microinjection technique.

[39] Sally L. Van Wert and James A Saunders. (1992) Plant Physiol., 99(2), 365-367.

[40] Paul Christou, Dennis McCabe (1992) Particle Gun Transformation of Crop Plants Using Electric Discharge (ACCELL ${ }^{T M}$ Technology), Published in Probe, 2 (2).

[41] Mohammed S. Al-Dosari and Xiang Gao. (2009) AAPS J., 11(4), 671-681.

[42] Maria Laura Immordino, Franco Dosio, and Luigi Cattel. (2006) Int $J$ Nanomedicine., 1(3), 297-315. 